\title{
Beyond the Qs in the polyglutamine diseases
}

\author{
Harry T. Orr \\ Departments of Laboratory Medicine and Pathology, Genetics, Cell Biology and Development, Biochemistry, Molecular \\ Biology and Biophysics, and Institute of Human Genetics, University of Minnesota, Minneapolis, Minnesota 55455, USA
}

It was 10 years ago that Kurt Fischbeck and his colleagues (La Spada et al. 1991) reported the identification of a novel mutational mechanism that altered the sequence of a protein: the addition of glutamines to a polyglutamine tract within the androgen receptor (AR). This mutation occurred in individuals affected with the motor neuron disease spinal and bulbar muscular atrophy (SBMA) or Kennedy's disease. With the cloning of the genes affected in Huntington disease (HD; Huntington's Disease Collaborative Research Group 1993) and spinocerebellar ataxia type 1 (SCA1; Orr et al. 1993), and subsequently with the identification of the basis for several other neurodegenerative diseases (for review, see Zoghbi and Orr 2000), it has become apparent that an expansion of a polyglutamine tract is the mutational mechanism underlying several neurodegenerative diseases. Currently, this group of disorders consists of eight diseases (Table 1). At the DNA level, the polyglutamine diseases are result from the expansion of an unstable CAG triplet repeat, placing the polyglutamine diseases within a broader class of inherited disorders, the unstable trinucleotide repeat diseases (Warren 1996).

With the identification of trinucleotide repeat and polyglutamine expansions as disease-causing mutational mechanisms, two general questions arose. What are the mechanisms for the repeat instability at the DNA level, and what are the effects of polyglutamine expansion at the protein level? A discussion of the genetic mechanism of repeat instability is beyond the scope of this review (see McMurray 1999). The purpose of this review is to summarize information that has accumulated with regard to the effects of polyglutamine expansion at the protein level. Focusing on SCA1, HD, and SCA6, an attempt will be made to identify important insights into the pathogenic mechanisms of these intriguing disorders.

One might envisage two possible mechanisms for the pathogenic effects of an expanded polyglutamine tract: (1) a mechanism dependent on an alteration of the function of the full-length protein, and (2) a mechanism that is largely dependent on the biochemical property of the

E-MAIL harry@lenti.med.umn.edu; FAX (612) 626-2600.

Article and publication are at www.genesdev.org/cgi/doi/10.1101/ gad.888401. polyglutamine tract itself regardless of the protein context in which it is located. In the first case, the altered toxic function of the protein could be a new function that is, for the most part, unrelated to the natural function of the protein. On the other hand, the alteration in function could be related to the normal function of the protein. If this latter scenario were to be the case, it would seem more likely that the pathogenic process would vary between the polyglutamine diseases, at least at their outset. In the second case, a mechanism essentially dependent on properties of the polyglutamine tract would more likely result in a common pathogenic mechanism among different diseases. Taking this scheme to an extreme would be a pathogenic mechanism centered around the generation of a toxic polyglutamine peptide. Which of these possibilities seems the more likely?

\section{Early genetic evidence}

Early genetic evidence argued for a common pathogenic mechanism centered around, and perhaps limited to, the polyglutamine tract. Mutant tracts of uninterrupted glutamine residues were found in genes encoding proteins with no homology to each other outside of the polyglutamine stretch and presumably with a variety of functions. Yet, in each case, the wild-type, unaffected alleles have typically been shown to contain fewer than 35 glutamine residues. However, the pathogenic alleles contain 39 or more residues in the glutamine tract (Table 1). For each disease, the pathogenic effect of the expanded polyglutamine tract is a gain-of-function dominant phenotype usually resulting in a late-onset, progressive neurological disorder (Zoghbi and Orr 2000). Regardless of the disease, the longer the polyglutamine tract, the more severe the disease and the earlier its age of onset.

Evidence that a polyglutamine tract itself can be toxic stems from studies with transgenic mice as well as transfected cell lines. Transgenic mice expressing a small fragment of the huntingtin protein with an expanded polyglutamine tract develop abnormal neurological signs and neuropathology (Managiarini et al. 1996). Similarly, a peptide containing essentially just the expanded polyglutamine tract from the SCA3 gene product is toxic to cerebellar Purkinje cells in transgenic mice (Ikeda et al. 
Table 1. Polyglutamine diseases

\begin{tabular}{|c|c|c|c|c|}
\hline \multirow[b]{2}{*}{ Disease } & \multirow[b]{2}{*}{ Protein } & \multirow{2}{*}{$\begin{array}{c}\text { Molecular } \\
\text { weight } \\
\text { (kD) }\end{array}$} & \multicolumn{2}{|c|}{$\begin{array}{l}\text { Polyglutamine } \\
\text { tract length }\end{array}$} \\
\hline & & & wild type & mutant \\
\hline SBMA & $\begin{array}{l}\text { androgen } \\
\text { receptor }\end{array}$ & 99 & $9-36$ & $38-62$ \\
\hline Huntington's & huntingtin & 348 & $6-35$ & $36-121$ \\
\hline DRPLA & antrophin-1 & 124 & $3-36$ & $49-88$ \\
\hline SCA-1 & ataxin-1 & 87 & $6-44$ & $40-82$ \\
\hline SCA-2 & ataxin-2 & 90 & $14-32$ & $33-77$ \\
\hline SCA-3 & ataxin-3 & 42 & $12-40$ & $55-86$ \\
\hline SCA-6 & $\begin{array}{l}\mathrm{P} / \mathrm{Q} \mathrm{Ca}^{2+} \\
\text { channel }\end{array}$ & $160-250$ & $4-18$ & $21-30$ \\
\hline SCA-7 & ataxin-7 & 95 & $7-17$ & $38-200$ \\
\hline
\end{tabular}

1996). Most notably, inserting a polyglutamine tract into the HPRT (hypoxanthine phosphoribosyl transferase) gene results in mice with a neurological phenotype very similar to that observed in mice expressing the mutant HD truncated protein (Ordway et al. 1997). There are also a number of studies demonstrating that expanded polyglutamine tracts within truncated proteins are more toxic to transfected cells than expanded tracts embedded in full-length proteins (for review, see Hackam et al. 1998). These studies imply that proteolytic cleavage is a necessary step in the initiation of polyglutamine disease.

\section{The aggregate hypothesis}

The association of aberrant deposits of a polyglutamine protein with neurological disease first burst into the literature in 1997, with reports of neuronal nuclear inclusions of a mutant huntingtin fragment in the brains of HD transgenic mice (Davies et al. 1997a) and within the brains of HD patients (DiFiglia et al. 1997). Very quickly, similar deposits of polyglutamine proteins were reported in neurons in SCA3 patient material (Paulson et al. 1997), and in neurons in SCA1 transgenic mice and patient material (Skinner et al. 1997). Subsequently, nuclear inclusions were reported in patient material for many of the other polyglutamine disorders, and it did not take long for the protein deposits to become the dominant force driving polyglutamine disease research (Davies et al. 1997b; Ross 1997; Perutz 1999). Without question, several aspects of the polyglutamine aggregates add strong support to their being central to the pathogenic mechanism. Aggregates or deposits of polyglutamine protein are a prominent pathological hallmark of most polyglutamine disorders. The aggregates are usually found within the nucleus but have been reported in the cytoplasm of neurons from HD patient material (DiFiglia et al. 1997). The time course for the formation of these aggregates either in animal or cellular models of pathology is consistent with them being causative. In cell culture systems, aggregate formation correlates with an increase susceptibility to cell death (Hackam et al. 1998). Furthermore, in vitro aggregation studies indicate that fragments of the HD protein with polyglutamine lengths within the pathological range, that is, longer than 40 , are insoluble whereas fragments carrying nonpathogenic repeat lengths are soluble (Scherzinger et al. 1997). Thus, coupled with indications that abnormal protein aggregation is the molecular basis for other neurodegenerative diseases, for example, Alzheimer's, Parkinson's, and prion diseases, the importance of protein deposits provides an attractive unifying pathogenic model for the polyglutamine diseases (Kaytor and Warren 1999).

\section{Cracks in the pestilent nature of polyglutamine aggregates?}

A suggestion that the polyglutamine aggregates may not be the basis for cell death seen in the polyglutamine diseases came from observations that the aggregates are not restricted in their presence to the cellular sites of pathology in SCA7 patient material. (Holmberg et al. 1998). Yet, like all of the earlier studies suggesting a pathogenic nature for the aggregates, this SCA7 study remains correlative. The first studies to examine directly the effect on disease of alteration of the aggregation status of a polyglutamine were published later in 1998. Using transfected cultured striatal cells expressing the amino-terminal fragment of the HD protein with an expanded polyglutamine Saudou et al. (1998) showed that exposure of these cells to conditions that reduced the formation of aggregates increases cell death. In a transgenic mouse model of SCA1 Purkinje cell disease, expression of the SCA1 gene product, ataxin-1, containing 77 glutamines, and a deletion of the self-association region results in ataxia and pathology in the absence of detectable aggregates of ataxin-1 (Klement et al. 1998). Furthermore, crossing a SCA1 disease-causing transgene (Burright et al. 1995) onto a murine genetic background lacking the expression of the ubiquitin E3 ligase Ube3A suppresses the formation of ataxin-1 aggregates. Yet, the progression of ataxin-1-induced pathology is enhanced considerably in the absence of Ube3A expression (Cummings et al. 1999). These reports provide strong evidence that the intranuclear aggregates of ataxin-1 and huntingtin may not be pernicious after all. Moreover, the results of the Saudou et al. (1998) and Cummings et al. (1999) studies indicate that the aggregates reflect a cellular mechanism that protects neurons from the toxic effects of a mutant polyglutamine protein. Thus, these observations argue that it is the soluble polyglutamine protein that is critical for triggering pathogenesis. Formation of aggregates may be a strategy by which the cell sequesters the misfolded polyglutamine protein thereby reducing its ability to associate with other cellular components.

\section{The ubiquitin-proteasome pathway and chaperones, the unfolding of polyglutamine aggregates}

Are other cellular defense mechanisms triggered in response to the expression of an expanded polyglutamine tract? It was recognized early on that nuclear polygluta- 
mine aggregates contain ubiquitin (Davies et al. 1997a; Paulson et al. 1997; Cummings et al. 1998). Covalent modification of proteins with ubiquitin and their subsequent degradation by the proteasomal apparatus is the major pathway by which cells remove improperly folded proteins (Wilkinson 2000). In addition to being positive for ubiquitin, cellular aggregates of polyglutamine proteins are found to be associated with components of the proteasomal machinery and molecular chaperones (Cummings et al. 1998). This observation supports the possibility that, as one might predict, proteins with an expanded polyglutamine tract exist in an abnormal conformation. Furthermore, manipulations of protein proteolytic and folding pathways might alter polyglutamine-induced pathological processes.

Evidence suggesting that a mutant polyglutamine protein is misfolded and triggers a cellular response came from the observation that the ataxin-1 nuclear aggregates in SCA1 patient neurons and transgenic mouse Purkinje cells stain positively with antibodies directed against the molecular chaperone protein HDJ-2/HSDJ (Cummings et al. 1998). Cell culture studies have gone on to indicate that protein misfolding and proteolysis can effect the cellular deposition of polyglutamine proteins. In HeLa cells expressing mutant ataxin-1, Cummings et al. (1998) showed that the expression of Hsp70 was induced, and that it was redistributed to the ataxin-1 nuclear aggregates. These investigators also reported that the ataxin-1 aggregation is decreased in HeLa cells cotransfected with ataxin-1 carrying 92 glutamines and HDJ-2/HSDJ. The effect of HDJ-2/HSDJ on ataxin-1 aggregate formation was dependent on the presence of the DnaJ-domain in HDJ-2/HSDJ. A similar result was obtained in HeLa cells expressing a mutant form of AR (Stenoien et al. 1999). Thus, molecular chaperones can modulate the aggregation of polyglutamine proteins, at least in a cell culture system.

In addition to the chaperones, polyglutamine aggregates are associated with components of the proteasomal apparatus (Cummings et al. 1998; Chai et al. 1999; Stenoien et al. 1999). Moreover, inhibiting proteasomal function leads to an increase in the formation of ataxin-3 (Chai et al. 1999) and ataxin-1 aggregates (Cummings et al. 1999) in transfected cells. These studies suggest that the proteasome is involved in the degradation of misfolded polyglutamine proteins. Evidence that a polyglutamine can be degraded by the ubiquitin-proteasome pathway (UPP) was reported for ataxin-1. Although ataxin-1 containing only two glutamines was polyubiquitinated to a level equal to ataxin-1 with 92 glutamines, and both forms were relatively resistant to proteasomal degradation, mutant ataxin-1 was more resistant to degradation (Cummings et al. 1999).

Recently, several laboratories have reported the establishment of polyglutamine-induced neurodegeneration in the fruitfly Drosophila melanogaster. These studies have provided direct evidence that molecular chaperones can suppress polyglutamine-induced neurotoxicity. Kazemi-Esfarjani and Benzer (2000) used a fly model expressing a glutamine tract with 127 residues to screen for suppressers of neurodegeneration. They identified two suppresser genes both containing chaperone-related J domains: dHDJ, which is homologous to human HSP 40/ HDJ1, and dTPR2, which is homologous to the human tetratricopeptide repeat protein 2. Using another approach, Chan et al. (2000) found that co-expression of dHdj1 in the eye of flies expressing truncated ataxin-3 with 78 glutamines suppresses degeneration. Yet, as assessed by light microscopy, chaperone suppression of polyglutamine disease in the fly is not accompanied by a similar decrease in the formation of aggregates in either study. These observations further raised the question of the pathogenic nature of polyglutamine aggregates. However, biochemical analyses of polyglutamine aggregates formed in the fly (Chan et al. 2000) or in vitro (Muchowski et al. 2000) in the presence of molecular chaperones have been interpreted as suggesting that chaperones act to direct polyglutamine from toxic relatively insoluble aggregates into nontoxic more soluble aggregates. Although the concept of toxic and nontoxic aggregates of polyglutamine proteins provides an explanation for the lack of toxicity in the presence of aggregates in the fly models, it is not clear how this concept would explain the demonstrations of polyglutamine-induced toxicity in the absence of aggregation (Klement et al. 1998; Saudou et al. 1998; Cummings et al 1999).

There is ample evidence that alterations in the cellular response to misfolded proteins can affect polyglutamine toxicity. Thus, the molecular chaperones and the proteasomal complex are likely to play an important role in protecting a neuron against the deleterious effects of an expanded polyglutamine tract. Perhaps the late onset typically seen in these disorders is due to these protective mechanisms becoming overwhelmed with time. It is also possible that cell-specific variation in the chaperone/proteasome response might contribute to the cellular specificity of pathology typical for each polyglutamine disease (Satyal et al. 2000). However, it remains unclear whether these defensive mechanisms are directly compromised by a misfolded polyglutamine protein and to what extent this would contribute to pathogenesis.

\section{Importance of protein context in polyglutamine-induced pathogenesis: lessons from three diseases}

In light of the above discussion, an examination of additional data obtained from analysis of three of the polyglutamine diseases (HD, SCA1, and SCA6) is quite revealing.

\section{Huntington disease}

$\mathrm{HD}$ is the most common and well-known of the polyglutamine diseases. HD consists of a triad of motor, cognitive, and emotional disturbances. The movement disorder in HD includes alterations in involuntary movements and abnormal voluntary movements. The most 
characteristic pathological feature of HD is neostriatal atrophy, particularly loss of the medium-sized spiny GABA neurons in the striatum. Other regions of the brain are also often affected. The whole brain often appears atrophic, and atrophy in the cortex is frequently seen.

In 1996, Managiarini et al. established transgenic mice in which the HD transgene consisted of a 1.9-kb fragment that included exon 1 with an expanded polyglutamine tract consisting of about $140-150$ repeats, as well as some $5^{\prime}$-flanking sequence from the HD gene. In the most extensively characterized transgenic line, R6/2, animals develop a progressive neurological phenotype by $8 \mathrm{wk}$ of age that included tremors, involuntary movements, and seizures, with death by 14 to 15 wk. Although these animals do have brains that are substantially smaller than their nontransgenic littermates, no significant neurodegeneration or other abnormalities have been identified. Thus, these animals do not show any signs of striatal neurodegeneration characteristic of HD. However, because they demonstrate a movement disorder somewhat like that seen in HD patients, these results suggest that $\mathrm{HD}$ could be caused by a truncated fragment of the huntingtin protein containing the polyglutamine tract. Perhaps in HD, a pathogenic fragment is generated from huntingtin by proteolysis.

Some support for the proteolytic hypothesis was obtained when the nuclear aggregates of huntingtin in patient material were examined with antibodies directed at different segments of huntingtin (DiFiglia et al. 1997). In autopsy material from one HD patient, the nuclear aggregates were reactive only to antibodies against the amino-terminal region of huntingtin. In addition, Western blot analysis of a brain extract indicated the presence of an amino-terminal fragment of huntingtin in the patient brain. Unfortunately, this latter observation has yet to be replicated.

Transgenic mice expressing the full-length $H D$ cDNA carrying 16,48 , or 89 CAG repeats have also been established (Reddy et al. 1998). In these mice, HD transgene expression is under the control of the cytomegalovirus promoter region. Thus, a wide pattern of transgene expression is seen. Mice expressing an HD cDNA with an expanded CAG repeat, 48 or 89 triplets, develop a progressive neurological abnormality consisting of altered limb clasping and generalized hyperactivity, unidirectional rotations, backflips, and excessive grooming. At 24 wk of age, these mice become hypoactive and die shortly thereafter. Neuronal loss is found in the striatum, a prominent site of pathology in HD patients, and in the hippocampus, thalamus, and cortex. Other areas of the brain with high levels of transgene expression show no signs of pathology, for example, the cerebellum. Nuclear inclusions of mutant huntingtin is detected in many areas of the brain, including Purkinje cells of the cerebellum. Contrary to suggestions that nuclear inclusions are pathogenic, the inclusions are less prominent in the striatum, where cell loss is the most extensive. Regardless of the cellular distribution of nuclear inclusions in the brain, this transgenic model of HD, express- ing full-length mutant huntingtin, shows a cellular pattern of pathology that is more typical of HD.

From a genetic perspective, the most accurate strategy for replicating HD in a mouse is to use gene targeting to insert an expanded, mutant CAG tract into the mouse huntingtin $(H d h)$ gene. Several groups have used such a knock-in approach to establish mouse models of HD. Mice carrying an insertion of 100 repeats in the $H d h$ gene demonstrate increased male aggression (Shelbourne et al. 1999). In contrast, insertions of from 90 to 111 repeats into $H d h$ result in no overt neurological alterations (Wheeler et al. 2000). In these mice, nuclear localization of normally cytoplasmic huntingtin is seen early on with nuclear inclusions forming much later. Very mild functional alterations have been reported with CAG insertions of 72 and 80 repeats (Levine et al. 1999; Usdin et al. 1999). Thus, Hdh knock-in mice with repeat tracts of around 100 units, which in humans results in a juvenile form of $\mathrm{HD}$, seem for the most part to have a subclinical phenotype. Mice with a longer 150 CAG repeat tract inserted into $H d h$ have been generated by gene targeting (Lin et al. 2001). These mice have a more severe neurological disease than mice with 100 repeats. Mice with 150 repeats in Hdh develop late-onset neurological abnormalities that include motor deficits and gait abnormalities. The behavioral alterations are associated with a striatal pathology consisting of reactive gliosis and the presence of neuronal intranuclear inclusions of huntingtin. Although neuronal loss in the striatum has yet to be seen in these animals, mice with 150 CAGs in the $H d h$ gene have a disease that more closely replicates HD.

Severity of the phenotype in HD transgenic mice seems to be dependent on several factors: the level of transgene expression, the length of the polyglutamine tract, and the protein context in which the polyglutamine tract is located. In all of the mouse models of HD, higher levels of transgene expression result in a more severe phenotype. For example, knock-in HD mice that are homozygous for the CAG insertion have a more severe phenotype regardless of the length of the expanded repeat tract (Wheeler et al. 2000; Lin et al. 2001). The importance of polyglutamine tract length as a determinant of disease severity has been shown in the YAC HD mice, the HD cDNA transgenics, and the CAG knock-in mice. Two lines of mice express the mutant huntingtin with 150 repeat units at a level comparable to endogenous huntingtin: the HD exon 1 transgenic mice (Managiarini et al. 1996), and the 150 repeat knock-in mice (Lin et al. 2001). The phenotype of the 150 repeat knock-in mice is not as severe as that seen in the HD exon 1 transgenic mice. These results suggest that a polyglutamine tract of a given length is more toxic when it appears in the context of a smaller protein. Although it seems that the toxic effects of a polyglutamine tract are masked by the full-length huntingtin protein, it is also apparent that the disease manifested by animals carrying a mutant polyglutamine tract is more similar to that seen in HD patients when the repeat is included within the full-length protein. 


\section{Spinocerebellar ataxia type 1}

SCA1 is an autosomal dominant neurodegenerative disease typically with mid-life onset characterized by motor symptoms in the absence of cognitive deficits. Death usually occurs between 10 and 15 years after the onset of symptoms. The clinical features of SCA1 vary depending on the stage of the disease, but typically include ataxia, dysarthria, and bulbar dysfunction. At the pathological level, the most frequent and severe alterations seen in SCA1 patients are losses of Purkinje cells in the cerebellar cortex and loss of neurons in the inferior olivary nuclei, the cerebellar dentate nuclei, and the red nuclei. Nuclei of the third, tenth, and twelfth cranial nerves also have variable involvement, with the hypoglossal nuclei being the most frequently and severely affected.

Burright et al. (1995) expressed full-length human SCA1 cDNAs with different numbers of CAG repeats in Purkinje cells using a Purkinje cell-specific promoter isolated from the Purkinje cell protein 2 gene (Pcp2/L7). The resulting mice express either a wild-type $S C A 1$ allele encoding 30 repeats (30Q) or an expanded allele encoding 82 repeats ( $82 \mathrm{Q})$ at high levels. Despite expressing high levels of wild-type ataxin-1, the 30Q mice show no signs of altered neurological function or Purkinje cell pathology and are indistinguishable from nontransgenic littermates. Adult ataxin-1-82Q mice develop severe ataxia and progressive Purkinje cell pathology (Burright et al. 1995; Clark et al. 1997). The earliest histologic abnormalities in the SCA1-82Q mice, detectable at postnatal day 25, are membranous cytoplasmic vacuoles. Loss of proximal dendritic arborization and dendritic spines becomes apparent at 5 wk, coinciding with the beginning of a mild impairment on the rotating rod. By the time the SCA1-82Q mice are observed to be ataxic by home cage behavior (12-15 wk), the dendritic arbor is mostly lost, the molecular layer is atrophied, and some heterotopic Purkinje cells have moved to the molecular layer. Cell loss becomes significant in the mice after $6 \mathrm{~m}$ of age, well beyond the onset of severe neurological deficits. Thus, the neurological alterations seen in these SCA1-82Q mice are not simply the result of Purkinje cell death. In contrast, mice with overexpression of a truncated polyglutamine fragment of the SCA3 gene product in Purkinje cells develop rapid cell loss leading to severe cerebellar atrophy as well as ataxia (Ikeda et al. 1996), a phenotype quite distinct from the progressive disease seen in SCA1 and SCA3 patients and in the SCA1 full-length transgenic mice.

Purkinje cell pathology seen in the SCA1-82Q transgenic mice is very similar to that seen in SCA1 patients, including the localization of mutant ataxin-1 to ubiquitin-positive nuclear inclusions (Skinner et al. 1997). These nuclear inclusions are present in a few Purkinje cells of SCA1-82Q mice beginning at 3-5 wk and are detectable in $90 \%$ of the Purkinje cells by 12 wk. An important question addressed with SCA1 transgenic mice was whether nuclear localization of mutant ataxin-1 was critical for disease. Klement et al. (1998) generated transgenic mice that expressed ataxin-1 pro- tein with 82 glutamines that had a mutated nuclear localization signal. In these mice, ataxin-1-82Q was distributed diffusely throughout the cytoplasm and showed no signs of the prominent nuclear localization of ataxin-1 seen in the initial SCA1-82Q mice. Although these mice expressed ataxin-1 in Purkinje cells at levels comparable to those observed in the original SCA1-82Q transgenic mice, they developed no Purkinje cell pathology or neurological dysfunction. Thus, nuclear localization of ataxin-1 is critical for pathogenesis.

The demonstration that mutation of the nuclear localization signal of ataxin-1, and thereby blockage of the entry of mutant ataxin-1 into the nucleus of Purkinje cells, completely abrogates the ability of the mutant protein to induce disease is an excellent example of how residues outside of the polyglutamine tract can have a role in disease. It is intriguing that mutant ataxin-2 (SCA2) is toxic when it is localized to the cytoplasm of Purkinje cells (Huynh et al. 2000) whereas cytoplasmic mutant ataxin-1 is not. These observations show that the subcellular site of polyglutamine toxicity can vary depending on the protein that contains the expanded polyglutamine tract.

Interactions between the polyglutamine protein and other cellular components is one biochemical property that would show a dependence on the subcellular localization and the protein context of a polyglutamine tract. In the case of ataxin-1, the leucine-rich nuclear protein (LANP) associates with ataxin-1 in a manner that decreases with increasing polyglutamine tract length (Matilla et al. 1997). Furthermore, LANP is localized to the nucleus and expressed at highest levels in Purkinje cells. Recently, it was shown that ataxin-1 can bind RNA and that this binding decreases with increasing number of polyglutamines (Yue et al. 2001). Thus, these are interesting molecular targets that might explain the importance of nuclear localization for the toxicity of mutant ataxin-1.

A recent and powerful application of Drosophila genetics to the study of polyglutamine pathogenesis was undertaken by Botas and colleagues (Fernandez-Funez et al. 2000). Using a fly model of SCA1, they performed a genetic screen for loci that modified SCA1-induced neurodegeneration. Several of the modifiers identified point further to the importance of protein folding and degradation for disease. Other modifiers reveal that genes whose products have a role in cytoplasmic/nuclear transport and RNA binding also affect SCA1-induced pathology in the fly. These studies confirm work performed in transgenic mice and are consistent with ataxin-1 having RNA-binding activity.

\section{Spinocerebellar ataxia type 6}

Like SCA1 and SCA2, neuronal loss in SCA6 consists of the prominent loss of Purkinje cells from the cerebellar cortex (Gomez et al. 1997). However, SCA6 is particularly intriguing in that it is one of only two diseases among the polyglutamine disorders for which the function of the protein is known (Table 1). The protein af- 
fected by the polyglutamine expansion in SCA6 is the $\alpha 1 \mathrm{~A}$ voltage-dependent calcium channel (Zhuchenko et al. 1997). In addition, the size range of the polyglutamine tract on mutant SCA6 alleles, 21-27 repeats, is much shorter than those found for the other diseases.

While aggregates of the $\alpha 1 \mathrm{~A}$-subunit have been detected in brains of SCA6 patients (Ishikawa et al. 1999), the polyglutamine expansion also alters the kinetic properties of the channel (Restituito et al. 2000). In Xenopus oocytes expressing $\alpha 1 \mathrm{~A}$-subunits with different lengths of polyglutamine and different $\beta$-subunits, $\alpha 1 \mathrm{~A}$-subunits with 30 polyglutamines have an impaired voltage-dependent activation and slowed inactivation rate only when expressed with the $\beta 4$-subunit. Thus, SCA6 could very likely be another channelopathy in which pathology might be due to an excessive entry of calcium into Purkinje cells. If so, this would be an excellent example where polyglutamine-induced pathology is directly related to the normal function of the protein and is dependent on its interaction with other cellular proteins, that is, the $\beta 4$-subunit. However, a question remains: Is SCA6 completely distinct from the other polyglutamine diseases because of the relative short length of its mutant alleles, or is SCA6 indicative that the altered function of a mutant polyglutamine protein directly reflects its normal function? In addition, it is possible that expansion of the polyglutamine tract in the SCA6 calcium channel, although altering channel function, induces disease by a completely distinct pathway. Consider the polyglutamine expansion in AR that causes SBMA or Kennedy's disease. There is evidence that this expansion in $\mathrm{AR}$ results in a partial disruption of AR function. For example, SBMA affected males have problems with fertility and present with enlarged breasts. Yet, the polyglutamine expansion in SBMA, although altering AR function, also causes a severe motor neuron disease. This latter aspect of SBMA is a phenotype not seen in XY individuals who have complete loss of AR function and have testicular feminization with no signs of a motor neuron disease. Thus, a polyglutamine expansion may alter normal function of a protein and induce disease by different mechanisms.

\section{Closing comments}

Since the identification of the first polyglutamine disease-causing mutation, a large body of data has been obtained that is beginning to direct our understanding of these neurodegenerative diseases. Expansion of the polyglutamine tract results in an altered conformation that triggers cellular responses, as well as associations with molecular chaperones and the proteasomal system, important for protection against the mutant protein. It appears that these protective responses eventually become overwhelmed, and the mutant protein is then sequestered into aggregates. Studies of SCA1 and HD models argue strongly that the aggregates are not pathogenic (Klement et al. 1998; Saudou et al. 1998). Rather, they serve a protective function (Saudou et al. 1998; Cummings et al. 1999). It is becoming increasingly clear that, although polyglutamine tracts themselves are very toxic, residues outside of the polyglutamine tract in each disease-causing protein have important roles in defining the course and specificity of disease. These residues participate in important cellular processes such as the subcellular localization of the polyglutamine protein and its interaction with other cellular molecules that very likely impact on disease progression. Much remains to be learned about the importance of the full-length protein in disease. If initiation and progression of each of the polyglutamine diseases ends up being as tightly linked to the normal function of the protein as has been suggested for SCA6 (Restituito et al. 2000), it becomes less likely that a common mechanism of pathogenesis will ensue. In this case, therapeutic efforts would best be directed at the enhancement of cellular protective measures.

\section{Acknowledgments}

The work from the author's laboratory was supported by grant NS22920 from NINDS/NIH.

\section{References}

Burright, E.N., Clark, H.B., Servadio, A., Matilla, T., Feddersen, R.M., Yunis, W.S., Duvick, L.A., Zoghbi, H.Y., and Orr, H.T. 1995. SCA1 transgenic mice: A model for neurodegeneration caused by an expanded CAG trinucleotide repeat. Cell 82: 937-948.

Chai, Y., Koppenhafer, S.L., Shoesmith, S.J., Perez, M.K., and Paulson, H.L. 1999. Evidence for proteasome involvement in polyglutamine disease: Localization to nuclear inclusions in SCA3/MJD and suppression of polyglutamine aggregation in vitro. Hum. Mol. Genet. 8: 673-682.

Chan, H.Y.E., Warrick, J.M., Gray-Board, G.L., Paulson, H.L., and Bonini, N.M. 2000. Mechanisms of chaperone suppression of polyglutamine disease: Selectivity, synergy and modulation of protein solubility in Drosophila. Hum. Mol. Genet. 9: 2811-2820.

Clark, H.B., Burrignt, E.N., Yunis, W.S., Larson, S., Wilcox, C., Hartman, B., Matilla, A., Zoghbi, H.Y., and Orr, H.T. 1997. Purkinje cell expression of a mutant allele of SCA1 in transgenic mice leads to disparate effects on motor behaviors, followed by a progressive cerebellar dysfunciton and histological alterations. J. Neurosci. 17: 7385-7395.

Cummings, C.J., Mancini, M.A., Antalffy, B., DeFranco, D.B., Orr, H.T., and Zoghbi, H.Y. 1998. Chaperone suppression of aggregation and altered subcellular proteasome localization imply protein misfolding in SCA1. Nat. Genet. 19: 148-154.

Cummings, C.J., Reinstein, E., Sun, Y., Antalffy, B., Jiang, Y.-H., Ciechanover, A., Orr, H.T., Beaudet, A.L., and Zoghbi, H.Y. 1999. Mutation of the E6-AP ubiquitin ligase reduces nuclear inclusion frequency while accelerating polyglutamine-induced pathology in SCA1 transgenic mice. Neuron 24: 879-892.

Davies, S.W., Turmaine, M., Cozens, B.A., DiFiglia, M., Sharp, A.H., Ross, C.A., Scherzinger, E., Wanker, E.E., Mangiarini, L., and Bates, G.P. 1997a. Formation of neuronal intranuclear inclusions underlies the neurological dysfunction in mice transgenic for the HD mutation. Cell 90: 537-548.

Davies, S.W., Beardsall, K., Turmaine, M., DiFiglia, M., Aronin, N., and Bates, G.P. 1997b. Are neuronal intranuclear inclu- 
sions the common neuropathology of triplet-repeat disorders with polyglutamine-repeat expansions? Lancet 351: 131-133.

DiFiglia, M., Sapp, E., Chase, K.O., Davies, S.W., Bates, G.P., Vonsattel, J.P., and Aronin, N. 1997. Aggregation in huntingtin in neuronal intranuclear inclusions and dystrophic neurites in brain. Science 277: 1990-1993.

Fernandez-Funez, P., Nino-Rosales, M.L., de Gouyon, B., She, W-C., Luchak, J.M., Martinez, P., Turiegano, E., Benito, J., Capovilla, M., Skinner, P.J., et al. 2000. Identification of genes that modify ataxin-1-induced neurodegeneration. $\mathrm{Na}$ ture 408: 101-106.

Gomez, C.M., Thompson, R., Gammack, J., Pearlman, S., Dobyns, W., Truwit, C., Zee, D., Clark, H.B., and Anderson, J.H. 1997. SCA6: Horizontal gaze-evoked and vertical nystagmus, Purkinje cell degeneration and variable age of onset. Ann. Neurol. 42: 933-950.

Hackam, A.S., Wellington, C.L., and Hayden, M.R. 1998. The fatal attraction of polyglutamine-containing proteins. Clin. Genet. 53 : 233-242.

Holmberg, M., Duyckaerts, C., Dürr, A., Cancel, G., Gourfinkel-An, I., Damier, P., Faucheux, B., Trottier, Y., Hirsch, E.C., Agid, Y., et al. 1998. Spinocerebellar ataxia type 7 (SCA7): A neurodegenerative disorder with neuronal intranuclear inclusions. Hum. Mol. Genet. 7: 913-918.

Huntington's Disease Collaborative Research Group. 1993. A novel gene containing a trinucleotide repeat that is unstable on Huntington's disease chromosomes. Cell 72: 971-983.

Huynh, D.P., Figuerosa, K., Hoang, N., and Pulst, S.-M. 2000. Nuclear localization or inclusion body formation of ataxin-2 are not necessary for SCA2 pathogenesis in mouse or human. Nat. Genet. 26: 44-50.

Ikeda, H., Yamaguchi, M., Sugai, S., Aze, Y., Narumiya, S., and Kakizukak, A. 1996. Expanded polyglutamine in the Machado-Joseph disease protein induces cell death in vitro and in vivo. Nat. Genet. 13: 196-202.

Ishikawa, K., Fujigasaki, H., Saegusa, H., Ohwada, K., Fujita, T., Iwamoto, H., Komatsuzaki, Y., Toru, S., Toriyama, H., Watanabe, M., et al. 1999. Abundant expression and cytoplasmic aggregations of alpha $1 \mathrm{~A}$ voltage-dependent calcium channel protein associated with neurodegeneration in spinocerebellar ataxia type 6. Hum. Mol. Genet. 8: 1185-1193.

Kaytor, M.D. and Warren, S.T. 1999. Aberrant protein deposition and neurological disease. J. Biol. Chem. 274: 3750737510.

Kazemi-Esfarjani, P. and Benzer, S. 2000. Genetic suppression of polyglutamine toxicity in Drosophila. Science 287: 18371840.

Klement, I.A., Skinner, P.J., Kaytor, M.D., Yi, H., Hersch, S.M., Clark, H.B., Zoghbi, H.Y., and Orr, H.T. 1998. Ataxin-1 nuclear localization and aggregation: Role in polyglutamineinduced disease in SCA1 transgenic mice. Cell 95: 41-53.

La Spada, A.R., Wilson, E.M., Lubahn, D.B., Harding, A.E., and Fischbeck, K.H. 1991. Androgen receptor gene mutations in $\mathrm{X}$-linked spinal and bulbar muscular atrophy. Nature 352: $77-79$

Levine, M.S., Klapstein, G.J., Koppel, A., Gruen, E., Cepeda, C., Vargas, M.E., Jokel, E.S., Carpenter, E.M., Zanjani, H., Hurst, R.E., et al. 1999. Enhanced sensitivity to N-methyl-D-aspartate receptor activation in transgenic and knockin mouse models of Huntington's disease. J. Neurosci. Res. 58: 515532 .

Lin, C.-H., Tallaksen-Greene, S.M., Chien, W.-M., Cearley, J.A., Jackson, W.S., Crouse, A.B., Ren, S., Li, X.-J., Albin, R.L., and Detloff, P.J. 2001. Neurological abnormalities in a knock-in mouse model of Huntington's disease. Hum. Mol. Genet. 10: $137-144$.
Managiarini, L., Sathasivam, K., Seller, M., Cozens, B., Harper, A., Hetherington, C., Lawton, M., Trottier, Y., Lehrach, H., Davies, S.W., et al. 1996. Exon 1 of the HD gene with an expanded CAG repeat is sufficient to cause a progressive neurological phenotype in transgenic mice. Cell 87: 493-506.

Matilla, A., Koshy, B.T., Cummings, C.J., Isobe, T., Orr, H.T., and Zoghbi, H.Y. 1997. The cerebellar leucine-rich acidic nuclear protein interacts with ataxin-1. Nature 389:974978.

McMurray, C.T. 1999. DNA secondary structure: A common and causitive factor for expansion in human disease. Proc. Natl. Acad. Sci. 96: 1823-1825.

Muchowski, P.J., Schaffar, G., Sittler, A., Wanker, E.E., HayerHartl, M.K., and Hartl, F.U. 2000. Hsp70 and Hsp40 chaperones can inhibit self-assembly of polyglutamine proteins into amyloid-like fibrils. Proc. Natl. Acad. Sci. 97: 78417846.

Orr, H.T., Chung, M.-Y., Banji, S., Kwiatkowski, Jr., T.J., Servadio, A., Beaudet, A.L., McCall, A.E., Duvick, L.A., Ranum, L.P.W., and Zoghbi, H.Y. 1993. Expansion of an unstable trinucleotide CAG repeat in spinocerebellar ataxia type 1 . Nat. Genet. 4: 221-226.

Ordway, J.M., Tallaksen-Greene, S., Gutekunst, C.A., Bernstein, E.M., Cearley, J.A.,Wiener, H.W., Dure, L.S., Lindsey, R., Hersch, S.M., Jope, R.S., et al. 1997. Ectopically expressed CAG repeats cause intranuclear inclusions and a progressive late onset neurological phenotype in the mouse. Cell 91: 753-63.

Paulson, H.L., Perez, M.K., Trottier, Y., Trojanowski, J.Q., Subramony, S.H., Das, S.S., Vig, P., Mandel, J.-L., Fischbeck, K.H., and Pittman, R.N. 1997. Intranuclear inclusions of expanded polyglutamine protein in spinocerebellar ataxia type 3. Neuron 19: 333-344.

Perutz, M.F. 1999. Glutamine repeats and neurodegenerative diseases: Molecular aspects. Trends Biochem. Sci. 24: 58-63.

Reddy, P.H., Williams, M., Charles, V., Garrett, L., PikeBuchanan, L., Whetsell, Jr., W.O., Miller, G., and Tagle, D.A. 1998. Behavioural abnormalities and selective neuronal loss in HD transgenic mice expressing mutated full-length HD cDNA. Nature Genet. 20: 198-202.

Restituito, S., Thompson, R.M., Eliet, J., Raike, R.S., Riedl, M., Charmet, P., and Gomez, C.M. 2000. The polyglutamine expansion in spinocerebellar ataxia type 6 causes a $\beta$ subunitspecific enhanced activation of P/Q-type calcium channels in Xenopus oocytes. J. Neurosci. 20: 6394-6403.

Ross, C.A. 1997. Intranuclear neuronal inclusions: A common pathogenic mechanism for glutamine-repeat neurodegenerative diseases? Neuron 10: 1147-1150.

Satyal, S.H., Schmidt, E., Kitagawa, K., Sondheimer, N., Lindquist, S., Kramer, J.M., and Morimoto, R.I. 2000. Polyglutamine aggregates alter protein folding homeostasis in Caenorhabditis elegans. Proc. Natl. Acad. Sci. 97: 5750-5755.

Saudou, F., Finkbeiner, S., Devys, D., and Greenberg, M.E. 1998. Huntingtin acts in the nucleus to induce apoptosis but death does not correlate with the formation of intranuclear inclusions. Cell 95: 55-66.

Scherzinger, E., Lurz, R., Turmaine, M., Managiarini, L., Hollenbach, B., Hasenbank, R., Bates, G.P., Davies, S.W., Lehrach, H., and Wanker, E.E. 1997. Huntingtin-encoded polyglutamine expansions form amyloid-like protein aggregates in vitro and in vivo. Cell 90: 549-58.

Shelbourne, P.F., Killeen, N., Hevner, R.F., Johston, H.M., Tecott, L., Lewandoski, M., Ennis, M., Ramierz, L., Li, Z., Iannicola, C., et al. 1999. A Huntington's disease CAG expansion at the mouse $H d h$ locus is unstable and associated with behavioural abnormalities in mice. Hum. Mol. Genet. 
8: $763-774$.

Skinner, P.J., Koshy, B., Klement, I.A., Helin, K., Servadio, A., Zoghbi, H.Y., and Orr, H.T. 1997. SCA1 pathogenesis involves alterations in nuclear matrix-associated structures. Nature 389: 971-974.

Stenoien, D.L., Cummings, C.J., Adams, H.P., Mancini, M.G., Patel, K., DeMartino, N., Marcelli, M., Weigel, N.L., and Mancini, M.A. 1999. Polyglutamine-expanded androgen receptors form aggregates that sequester heat shock proteins, proteasome components and SRC-1, and are suppressed by the HDJ-2 chaperone. Hum. Mol. Genet. 8: 731-741.

Usdin, M.T., Shelbourne, P.F., Myers, R.M., and Madison, D.V. 1999. Impaired synaptic plasticity in mice carrying the Huntington's disease mutation. Hum. Mol. Genet. 8: 839-846.

Warren, S.T. 1996. The expanding world of trinucleotide repeats. Science 271: 1374-1375.

Wheeler, V.C., White, J.K., Gutekunst, C.A., Vrbanac, V., Weaver, M., Li, X.J., Li, S.H., Yi, H., Vonsattel, J.P., Guesella, J.F., et al. 2000. Long glutamine tracts cause nuclear localization of a novel form of huntingtin in medium spiny neurons in Hdh(Q92) and Hdh(Q111) knock-in mice. Hum. Mol. Genet. 9: 503-513.

Wilkinson, K.D. 2000. Ubiquitination and deubiquitination: Targeting of proteins for degradation by the proteasome. Sem. Cell Develop. Bio. 11: 141-148.

Yue, S., Serra, H.G., Zoghbi, H.Y., and Orr, H.T. 2001. The spinocerebellar ataxia type 1 protein, ataxin-1, has RNAbinding activity that is inversely affected by the length of its polyglutamine tract. Hum. Mol. Genet. 10: 25-30.

Zhuchenko, O., Bailey, J. Bonnen, P., Ashizawa, T., Stockton, D.W., Amos, C., Dobyns, W.B., Subramony, S.H., Zoghbi, H.Y., and Lee, C.C. 1997. Autosomal dominant cerebellar ataxia (SCA6) associated with small polyglutamine expansions in the $\alpha 1 \mathrm{~A}$-voltage-dependent calcium channel. Nat. Genet. 15: 62-69.

Zoghbi, H.Y. and Orr, H.T. 2000. Glutamine repeats and neurodegeneration. Annu. Rev. Neurosci. 23: 217-247. 


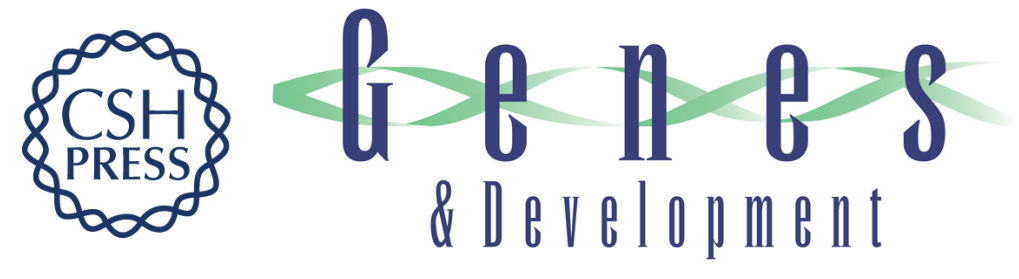

\title{
Beyond the Qs in the polyglutamine diseases
}

\author{
Harry T. Orr
}

Genes Dev. 2001, 15:

Access the most recent version at doi:10.1101/gad.888401

References This article cites 45 articles, 8 of which can be accessed free at: http://genesdev.cshlp.org/content/15/8/925.full.html\#ref-list-1

\section{License}

Email Alerting

Receive free email alerts when new articles cite this article - sign up in the box at the top Service right corner of the article or click here.

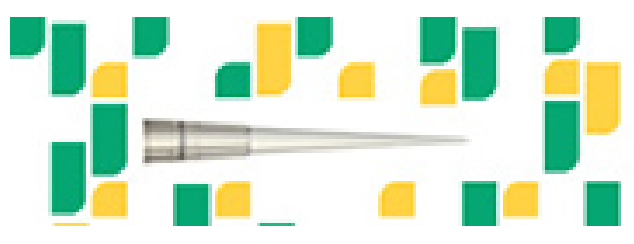

Focused on your science. 